\title{
PREPARATION AND EVALUATION OF SOME PIGMENTS TO BE USED IN AIRCRAFT PAINTS
}

M.M.EI-Wakil,M.G.Soliman and M.Sh.Fayed

\section{ABSTRACT:}

Pigments are widely used in commercial compositions of plastics, rubbers, ceramics, printing inks, cosmetics and paints. The paints as surface coating compositions can be decorative or protective. In the field of aircraft, the outside finish must also protect against fluctuation of temperature, intense ultraviolet radiation and impact with raindrops at high velocity.

In this work a series of yellow to buff and blue to green plgments were prepared by the calcination process at $1100 \mathrm{C}$. Their spectral characteristics were measured and the relation between the absolute spectral data and wavelength of the incident radiation in the range of 400-1100 $\mathrm{nm}$ were given. The effect of percentage of pigment composition and the time of calcination were shown. The spectral data were measured for the pigments in powder and film forms. A discussion on the use of such inorganic pigments in the fleld of aircraft was reported. 


\section{IHTFODUSTI DH: :-}

Faint $i=$ a fluid compoition shich, when spread over a surface in the form af $\Xi$ thin layer, will form a solid, adherent and cotesive film. This fluid paint contains three major ingredients: pigment, binder and thinner. In addition to these major ingredients, paint may contain small quantities of other components ajled additives such as: driers, antisettling agent: Fungicides and others [1].

Figriente are chemical compounds, either organic or inorganic,

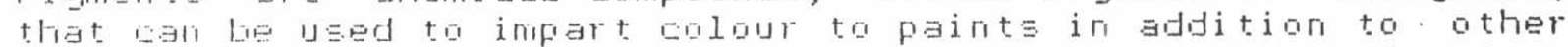
yarious commeraial. materials such as : ceramics, plastics, colletias and firinting inks [2].

Figrient a are and remain insoluble when applied in different surfae cojirge. They extitit one ar more of three basic functing: aptical, frotective and reinforaing ones. Colour, oracity arid gloss are the most attractirg optical functions. Weather statility, surface hardness, adhesion, flexibility and aorroion prevention can be considered as their partiaipation in the protective furction. The perfect selection of the pigment and the level of pigmentaticn can affect-to larger extent- the film's cohesiveness, elasticity, hardness and abraison resistance which aid in the reinforcenent of the surface film $[3,4]$.

The performance of certain pigment depends on the pigment-binder syeteri, its refractive index, particle shafe and size distribution, and the efficiency of its dispersion in the MEdi unim[ 5$]$.

In military applications, the surface coating compositions must: al 0 funcion to harmonize the object with.each type of the expected aperation ground, so that it cannot be easily detected Ly yarious heang of observation and reconnaiseance. These means act i different ranges of electromagnetic rays from the visible, near infrared to the far infrared rays. This main criterion is added to the great statility of colour, frotection against arrogin, facility of the durability of all these properties in the field service of yariatle humidity, temperature and nind $[5,7]$.

In the presented work, some selected figrients of colours yellow to bufr antulue to green were prepared and evaluated by their absolute Efectral data in the wavelength range of 400 to $1150 \mathrm{rm}$.

\section{EXPEFIIIEITHL}

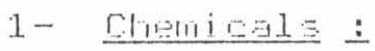

All chemicals used in the experimental work were of the laboratory grade. They were: Chromium oxide green (Bakin), antimony tri sulphide (Morgan), calcium carbonate (UEe); ti tanium dioxide (Eakin); cobaltous ahloride (Prolabo) and aluminium oxide (EDH). 
was sieved into tho frations of different grain size. The smaller grain size showed smaller reflectance throgh the total range of the wavelength, compared with that of the greater one.

These prepared compositions were relatively opaque and have good hiding power, and can be used to match the desert surroundings.

In the second group of cobalt aluminate blues, the main pigment was prepared from the blending of cobaltous chloride and aluminium oxide with molar ratio of $1: 1$ which was shown in Fig.4. The reflectance curve showed a rapid increase in the curve after atout 650 nin. The reflectance in the near infrared region from 750 nii to 950 nim ranged between 60 to $65 \%$ and the maximum in the near infrared was reached at about 900 nm.

The addition of chromium oxide green to the last composition and its effect or the shape of the spectral curve was shown in Fig. 5. The reflectance curve was modified with the maximum ahifted to bbout 975 rm. The effect of calcination time was shoun in the sane figure where as the time was raised from 1,5 hrs 103 hrs the reflectance was increased by about $10 \%$ all over the near infrared range with a relatively smaller effect in the yisible range. These type of pigments can be used to match the agricultural surroundings.

In the field of aircraft application, it is well known that subsonic types of aircraft have a basic structure of aluminium alloy with high-tensile steel and magnesium alloys for. certain specialized parts. The best selection of the binder system for such structural alloyis the epoxy - polyamide systern in both primer and finish. This selection of binder system and the last mentioned $\mathrm{Figments}$ was to resist the wide fluctuations of temperature; intense ultrawiolet radiation at high altitudes, arid inpac: with raindrops at high velocity. This system of pigment - Linder is also resistant to fuels and to hydraulic, brake and de-icing fluids. The temperature fluctuations can result in condensation af appreciable amounts of water inside the outer skin. This can boil at the temperature reached by the skin of some aircraft and $i s$ a potent source of corrosion. So this an be avoided by such selection of pigment-binder system in adjiton lo the use of strontium chromate pigment in the primer.

Another modification in the binder is the use of polyurethane based on aliphatic isocyanate in the finish and the primer of epoxy-polyamide type, which give good gloss-retention, colour and chemical resistance.

With high-speed aircraft, the exposed temperatures are higher, the same system can be used. Acrylics being characterized by showing good colour stability at such temperatures, can be applied.

Two-pack epoxies are not favoured for the exterior of modern aircraft. They chalk rapidly at high altitudes where the
ultrayiolet raidation is more intense. than at lower levels. The interior alloy surfaces can be finished in two-pack epoxy enamel 
Thi bo Eeloted groupe of the pignents were frepared from the orrejorning chenical compounds capable of yielding the metal arides though a calciration froces at high temperature. The exat amount of each compond was accurately. weighed. The anmourde mere, then, intimately blended tagether in a cortrifugal ball mill for fem minutes and after that the mixture 1. a aloined at a temperature of 11000 for two different periods of time : 1,5 and 3 hours. The pigment was graund to a fine
powder and evaluated by its absolute spectral data in the 1avelength range of incident radiation from $400 \mathrm{to} 1150 \mathrm{~nm}$. The effect of alcination time, figment composition and particle size gi stribution mere rieasured.

\section{S- In}

The atoulute epectral data were measured ty an optronic Infrared Spectroradiometer Model. 746 equipped with an IBM computer and Hemelett plotter. The rixing and puluerizing of the pigment composilin were executed by a Retsch centrifugal Ball Mill of type 31 . The calcination was carried out in a thermostated Mufle furnace Model NAEER 2004 Eremen acting up to $12000 \mathrm{C}$.

\section{FEEILTS AHD DISCUSSIONS :} Two dirrerent groups of pigments were selected to be used in
durabje boatings, designed for exteriar applications over a yariety of substrate materials such as steel, aluminium and hardbord, and carrying long-term waranties. These pigments can be $[0 n \equiv i j e r$ d as alternatives after the restrictions and concerns ouer the use of the toxis lead-based yellow and a adrium - based F. i grient:

Three tha groups are of the types chrome-titanate buffs and arbal jluninate tlues. Eecause they are prepared wia the calainator process yielding a arystals of mixed oxides, these pighente will be chemically inert that are not attacked by acids or alkalie, completely statele and heat resistant.

In the group of chrom-titanate buffs, the spectral curves shown ir Fig. 1 indiates the effect of the composition of iritial ingredients or the absolute spectral data, where in one Grrposition titanium oxide was tuended with a mixture of chrome green and antimony trisulfide. In the other, calcium carbonate i.j added to the mixture before caloination. There was no appreciatie alıage in the reflectance af these two compositions with the tested ratio.

In Fig. 2 , the effect of calcination time of the previous composition yes shown at 1 , 5 and 3 hrs. The spectral reflectance showed a small increase all guer the range in the pigment of a alculatjon time 3 hre, compared with that of 1,5 hrs. The effect of ihe particle size was ghom in Fig. 3 . The prepared 
after the urface pretreatment and priming.

\section{COHCLUSION :}

The eelected groups of pigments can be widely used in the surface coating of airoraft. The two groups of pigments can match both the desert and the agricultural surroundings. They are resistant to high temperature, humidity and all chemicals since they ate prepared Via the calcination process. There is a wide range of colours that can be ottained by a slight change in the initial ingredients. These colours can be described by their specific epectral curves in the visible to the near infrared region of electromagnetic daves.

\section{FEFEREIICES:}

(1) Fothenterg, B.B., : "Faint Additives Recent Developments", Noges Data Corporation, 1978.

(2) Kirk 0uhmer : "Encyolopedia of Chenical Technology", John Hiley, 1981 .

(3) Morgane, W.M., : "Outlines of Paint Technology, Vol. 2, John Wiley, 1984.

(4) Konstandt, F., : "Organic Coatings, Froperties and Evaluation", Chem. Publish. Co., 1985.

(5) Fatton, T.C., : "Faint Flow and pigment Dispersion", John Wiley, 1979 .

(6) Dil anid Colour Chenicsts Association, Australia : "Surface Coating " Vol 1 and 2 ; 2nd Ed., Chapman, 1983 and 1984.

(7) Hlunt F.W.G., : Measuring Colour", Ellis Horwood, 1937. 


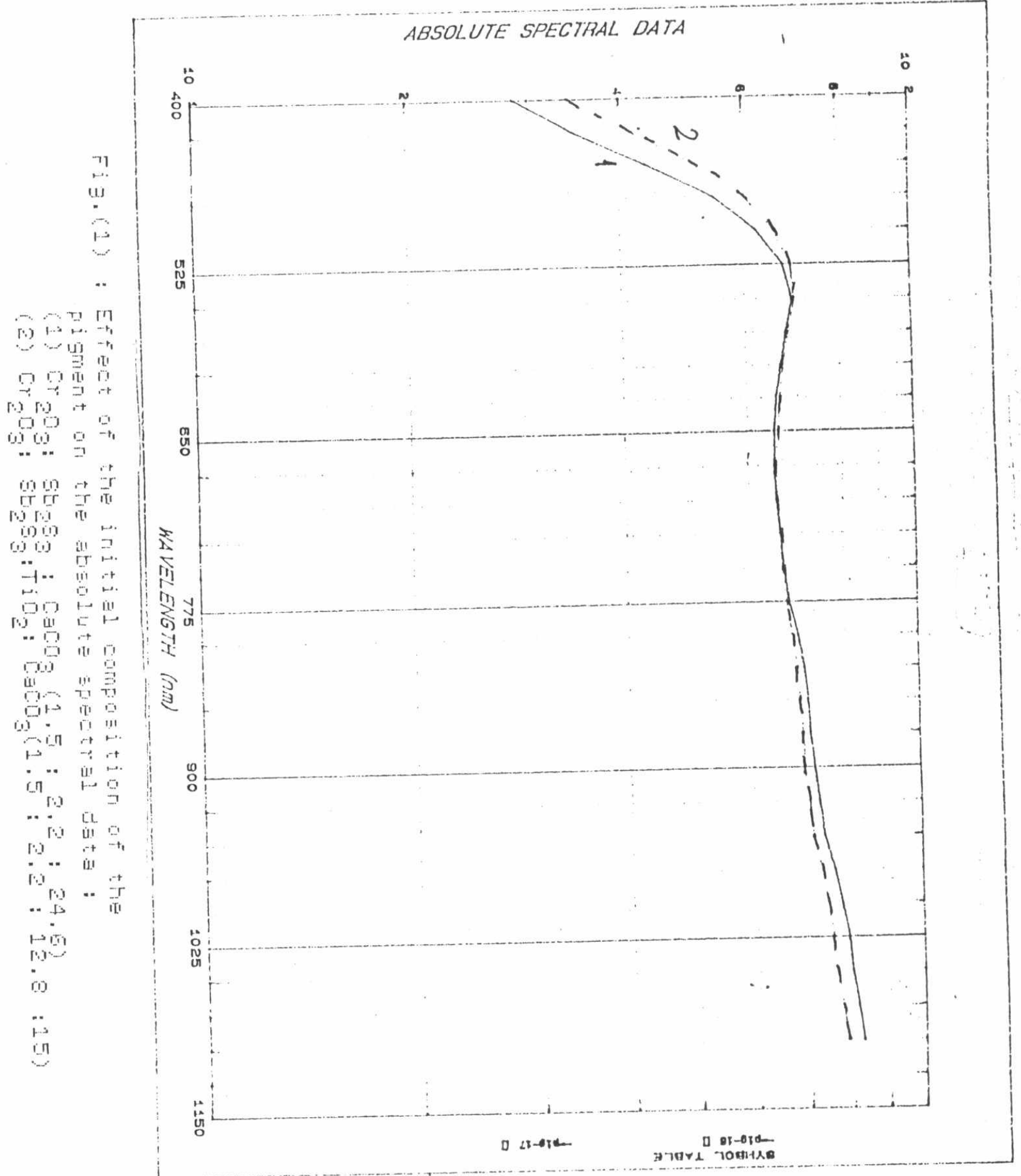




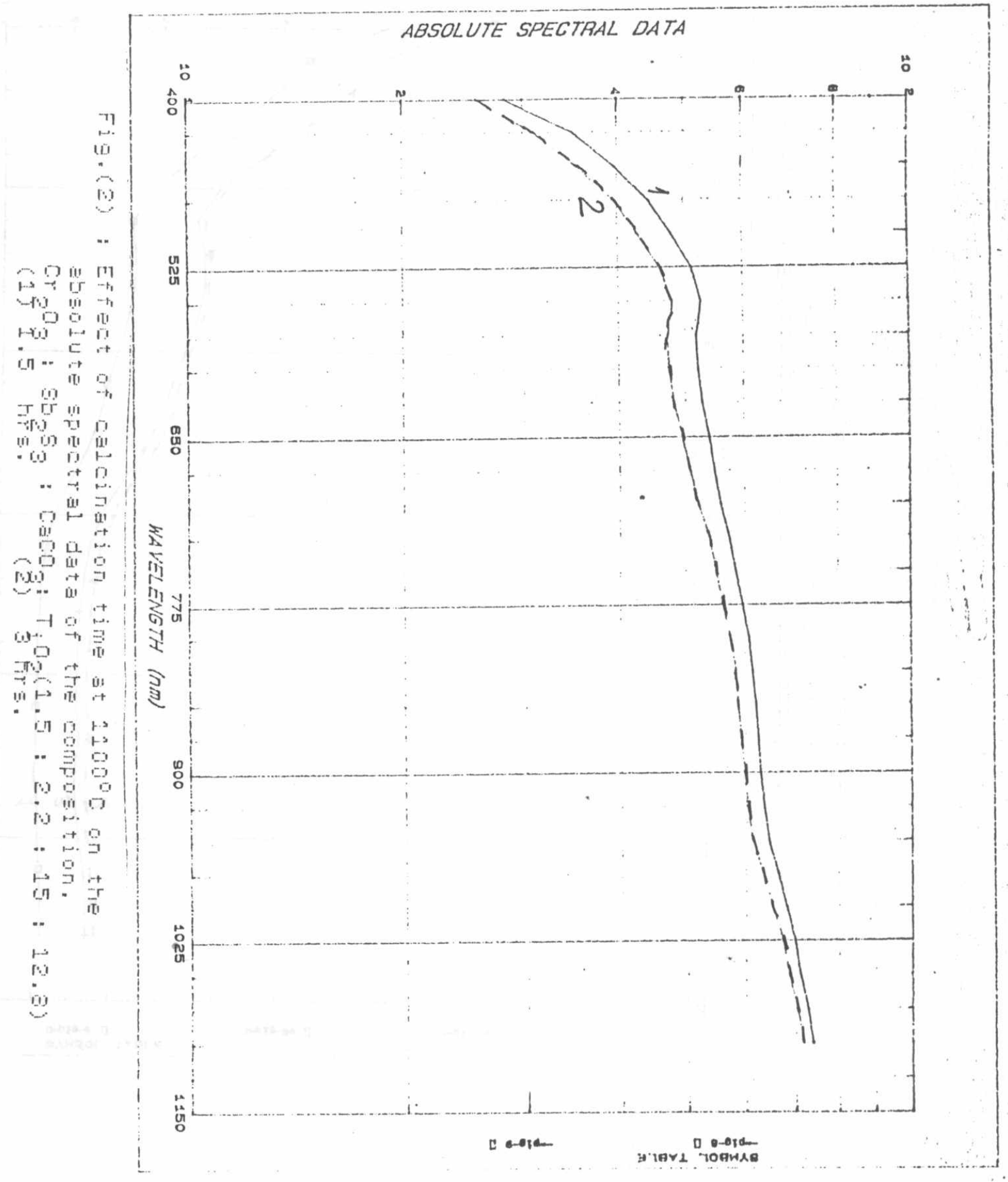




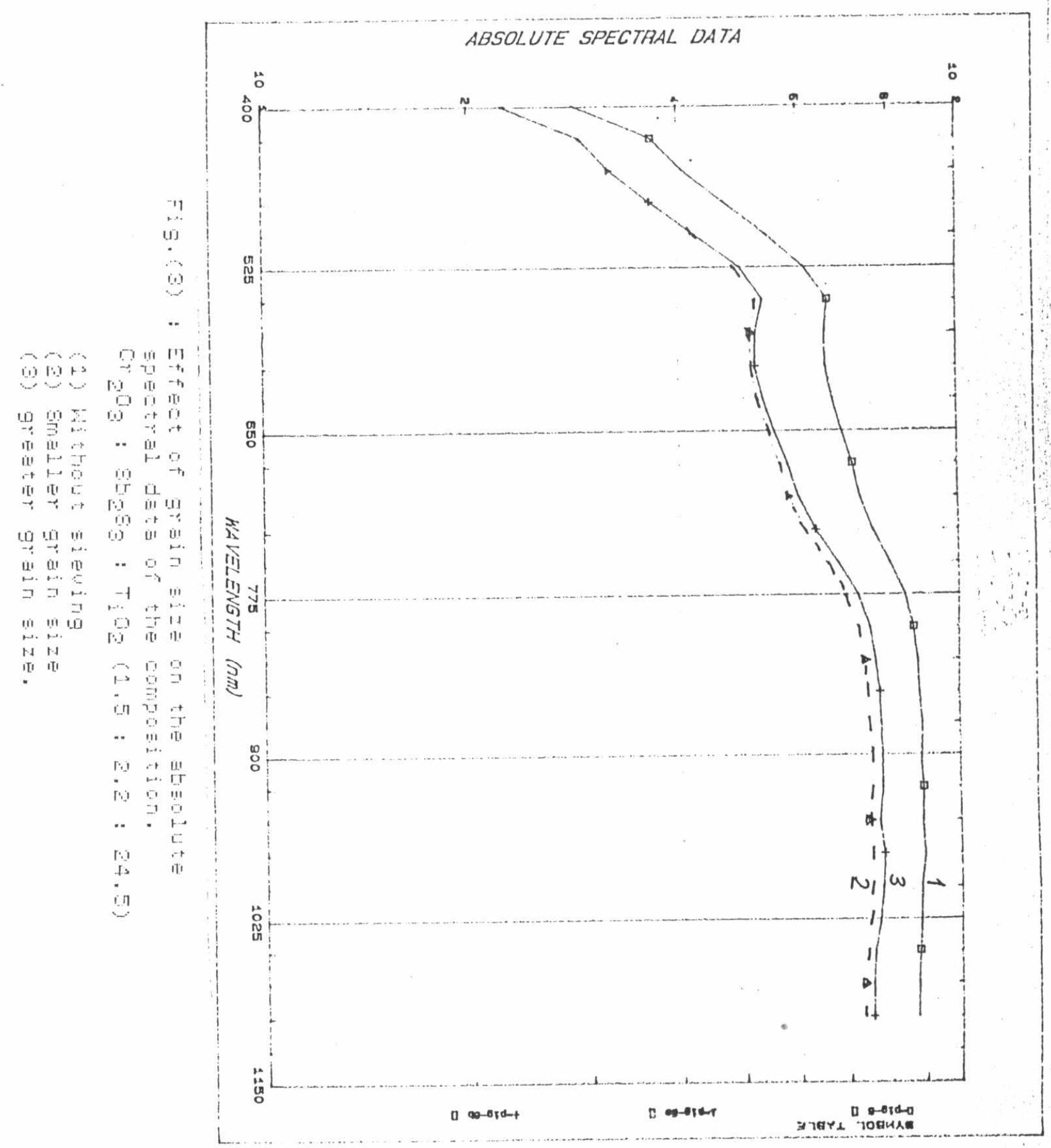




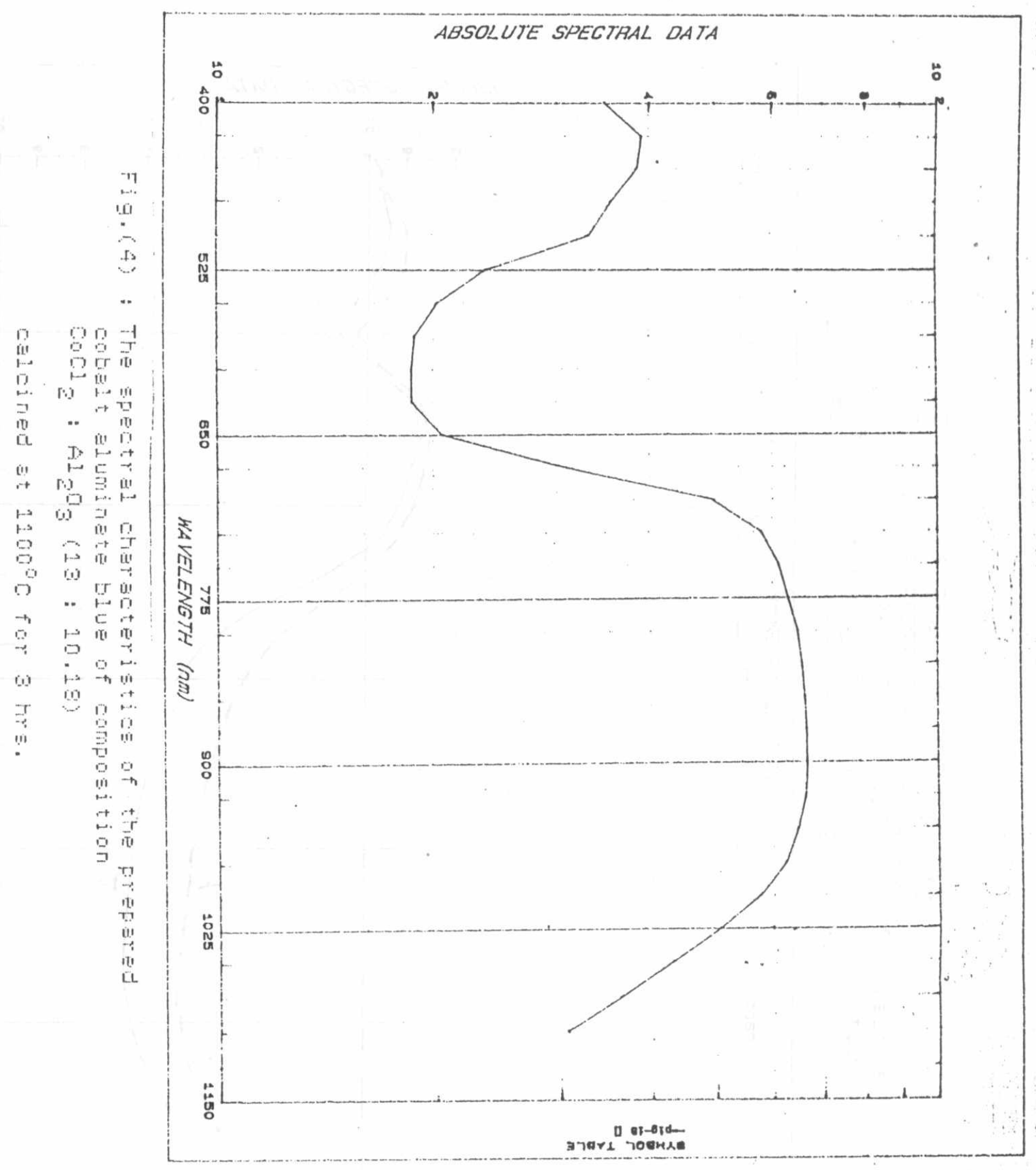




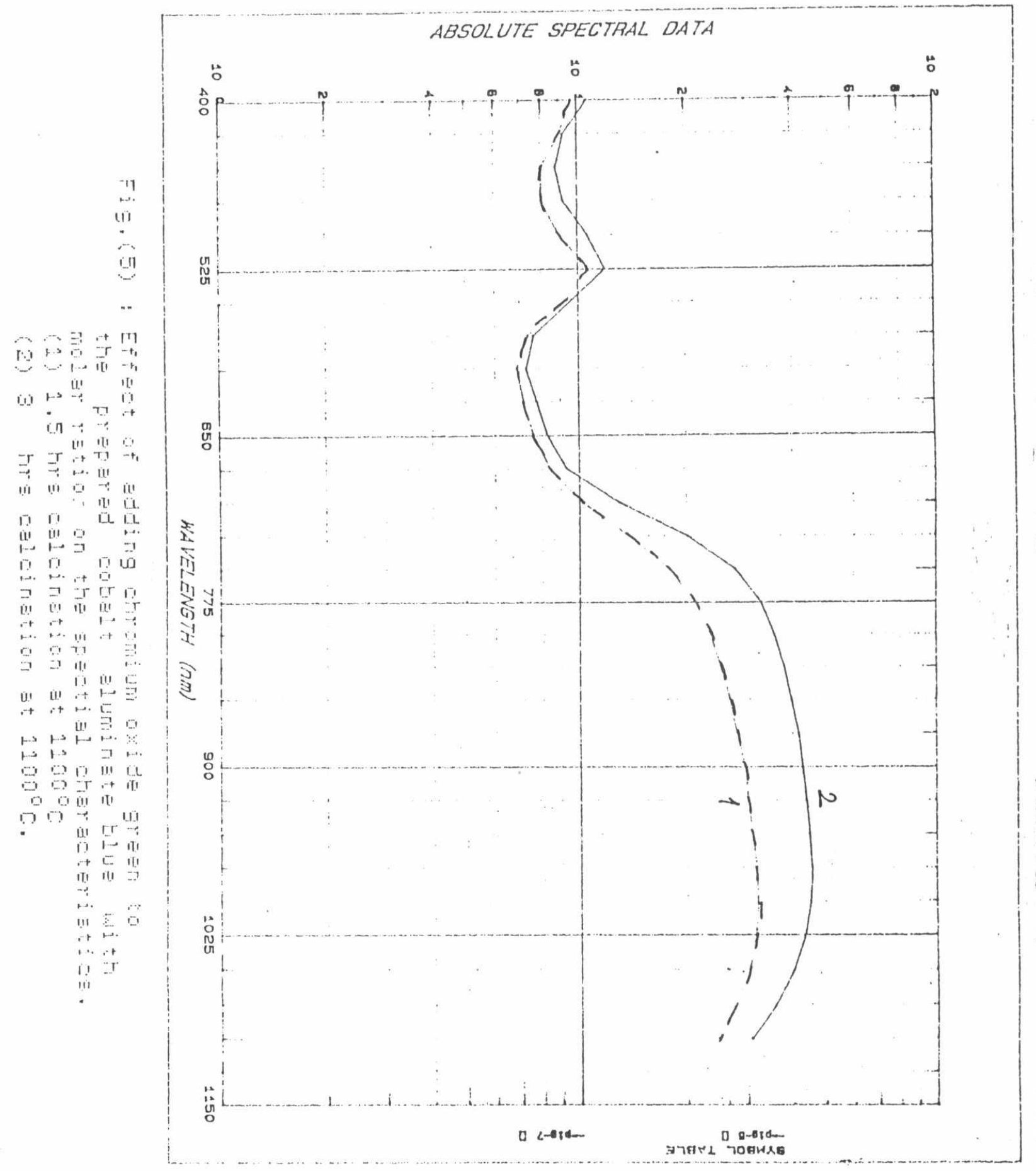

